\title{
SWIFT J2058.4+0516: DISCOVERY OF A POSSIBLE SECOND RELATIVISTIC TIDAL DISRUPTION FLARE?
}

\author{
S. Bradley Cenko ${ }^{1}$, Hans A. Krimm ${ }^{2,3}$, Assaf Horesh ${ }^{4}$, Arne Rau ${ }^{5}$, Dale A. Frail ${ }^{6}$, Jamie A. Kennea ${ }^{7}$, \\ Andrew J. Levan ${ }^{8}$, Stephen T. Holland ${ }^{9}$, Nathaniel R. Butler ${ }^{1,12}$, Robert M. Quimby ${ }^{4}$, Joshua S. Bloom ${ }^{1}$, \\ Alexei V. Filippenko ${ }^{1}$, Avishay Gal-Yam ${ }^{10}$, Jochen Greiner ${ }^{5}$, S. R. Kulkarni ${ }^{4}$, Eran O. Ofek ${ }^{4}$, Felipe Olivares E. ${ }^{5}$, \\ Patricia Schady ${ }^{5}$, JefFrey M. Silverman ${ }^{1}$, Nial R. TANVir ${ }^{11}$, and Dong Xu ${ }^{10}$ \\ ${ }^{1}$ Department of Astronomy, University of California, Berkeley, CA 94720-3411, USA; cenko@ astro.berkeley.edu \\ ${ }^{2}$ CRESST and NASA Goddard Space Flight Center, Greenbelt, MD 20771, USA \\ ${ }^{3}$ Universities Space Research Association, 10211 Wincopin Circle, Suite 500, Columbia, MD 21044, USA \\ ${ }^{4}$ Division of Physics, Mathematics, and Astronomy, California Institute of Technology, Pasadena, CA 91125, USA \\ ${ }^{5}$ Max-Planck Institute for Extraterrestrial Physics, Giessenbachstrasse 1, Garching 85748, Germany \\ ${ }^{6}$ National Radio Astronomy Observatory, P.O. Box 0, Socorro, NM 87801, USA \\ ${ }^{7}$ Department of Astronomy \& Astrophysics, The Pennsylvania State University, 525 Davey Laboratory, University Park, PA 16802, USA \\ ${ }^{8}$ Department of Physics, University of Warwick, Coventry CV4 7AL, UK \\ ${ }^{9}$ Space Telescope Science Institute, 3700 San Martin Drive, Baltimore, MD 21218, USA \\ ${ }^{10}$ Department of Particle Physics and Astrophysics, Faculty of Physics, The Weizmann Institute of Science, Rehovot 76100, Israel \\ ${ }^{11}$ Department of Physics and Astronomy, University of Leicester, University Road, Leicester LE1 7RH, UK \\ Received 2011 July 26; accepted 2012 April 23; published 2012 June 15
}

\begin{abstract}
We report the discovery by the Swift hard X-ray monitor of the transient source Swift J2058.4+0516 ( $\mathrm{Sw} \mathrm{J} 2058+05)$. Our multi-wavelength follow-up campaign uncovered a long-lived (duration $\gtrsim$ months), luminous X-ray $\left(L_{\mathrm{X} \text {,iso }} \approx 3 \times 10^{47} \mathrm{erg} \mathrm{s}^{-1}\right)$ and radio $\left(\nu L_{\nu, \text { iso }} \approx 10^{42} \mathrm{erg} \mathrm{s}^{-1}\right)$ counterpart. The associated optical emission, however, from which we measure a redshift of 1.1853 , is relatively faint, and this is not due to a large amount of dust extinction in the host galaxy. Based on numerous similarities with the recently discovered GRB 110328A/Swift J164449.3+573451 (Sw J1644+57), we suggest that Sw J2058+05 may be the second member of a new class of relativistic outbursts resulting from the tidal disruption of a star by a supermassive black hole. If so, the relative rarity of these sources (compared with the expected rate of tidal disruptions) implies that either these outflows are extremely narrowly collimated $\left(\theta<1^{\circ}\right)$ or only a small fraction of tidal disruptions generate relativistic ejecta. Analogous to the case of long-duration gamma-ray bursts and core-collapse supernovae, we speculate that rapid spin of the black hole may be a necessary condition to generate the relativistic component. Alternatively, if powered by gas accretion (i.e., an active galactic nucleus (AGN)), Sw J2058+05 would seem to represent a new mode of variability in these sources, as the observed properties appear largely inconsistent with known classes of AGNs capable of generating relativistic jets (blazars, narrow-line Seyfert 1 galaxies).
\end{abstract}

Key words: accretion, accretion disks - black hole physics - galaxies: nuclei - X-rays: bursts - X-rays: individual (Sw J1644+57)

Online-only material: color figures

\section{INTRODUCTION}

The recent discovery of the transient source GRB 110328A/ Swift J164449.3+573451 ( $\mathrm{Sw} \mathrm{J1644+57)} \mathrm{has} \mathrm{unveiled} \mathrm{an} \mathrm{en-}$ tirely new class of high-energy outbursts (Levan et al. 2011; Burrows et al. 2011; Zauderer et al. 2011). Like long-duration gamma-ray bursts (GRBs), the outburst was believed to mark the birth of a relativistic jet, generating luminous X-ray and radio emission. However, the central engine powering Sw J1644+57 was the supermassive $\left(M_{\mathrm{BH}} \lesssim 10^{7} M_{\odot}\right)$ black hole in the nucleus of an otherwise normal (i.e., nonactive) galaxy (Bloom et al. 2011). While not conclusive, the observed emission may result from the tidal disruption (Rees 1988) of a star (or white dwarf; Krolik \& Piran 2011) passing too close to the central black hole (Bloom et al. 2011; Burrows et al. 2011; Zauderer et al. 2011; Cannizzo et al. 2011). A number of tidal disruption flare (TDF) candidates have been previously identified at longer wavelengths (e.g., Renzini et al. 1995; Bade et al. 1996; Komossa \& Greiner 1999; Greiner et al. 2000; Donley et al. 2002; Gezari et al. 2006, 2008, 2009; Esquej et al. 2007, 2008;

\footnotetext{
12 Einstein Fellow.
}

Maksym et al. 2010; van Velzen et al. 2011; Cenko et al. 2012). But the robust inference of a newly born relativistic jet, and the insights into the accretion and jet-formation processes provided therein, clearly distinguishes $\mathrm{Sw} \mathrm{J} 1644+57$ from previous TDF candidates.

In this work, we report on a recently discovered high-energy transient, Swift J2058.4+0516 (Sw J2058+05). Remarkably, Sw $\mathrm{J} 2058+05$ shares many of the properties that made Sw J1644+57 such an exceptional event, in particular (1) a long-lived (duration $\gtrsim$ months), super-Eddington $\left(L_{\mathrm{X}, \text { iso }} \approx 3 \times 10^{47} \mathrm{erg} \mathrm{s}^{-1}\right.$ ) $\mathrm{X}$-ray outburst; (2) a luminous radio counterpart, indicating the presence of relativistic $(\Gamma \gtrsim 2)$ ejecta; and (3) relatively faint $\left(M_{U} \approx-22.7 \mathrm{mag}\right)$ optical emission. If future observations are able to establish an astrometric association with the nucleus of the redshift $z=1.1853$ host galaxy and also continue to suggest that this galaxy does not harbor an active galactic nucleus (AGN), we may have identified a second member of this new class of relativistic TDFs. Alternatively, several classes of AGNs are known to generate relativistic jets (blazars, narrowline Seyfert 1 [NLS1] galaxies). If Sw J2058+05 is shown to result from an AGN (e.g., via repeated outbursts over the coming years), we would have uncovered yet another unique example 


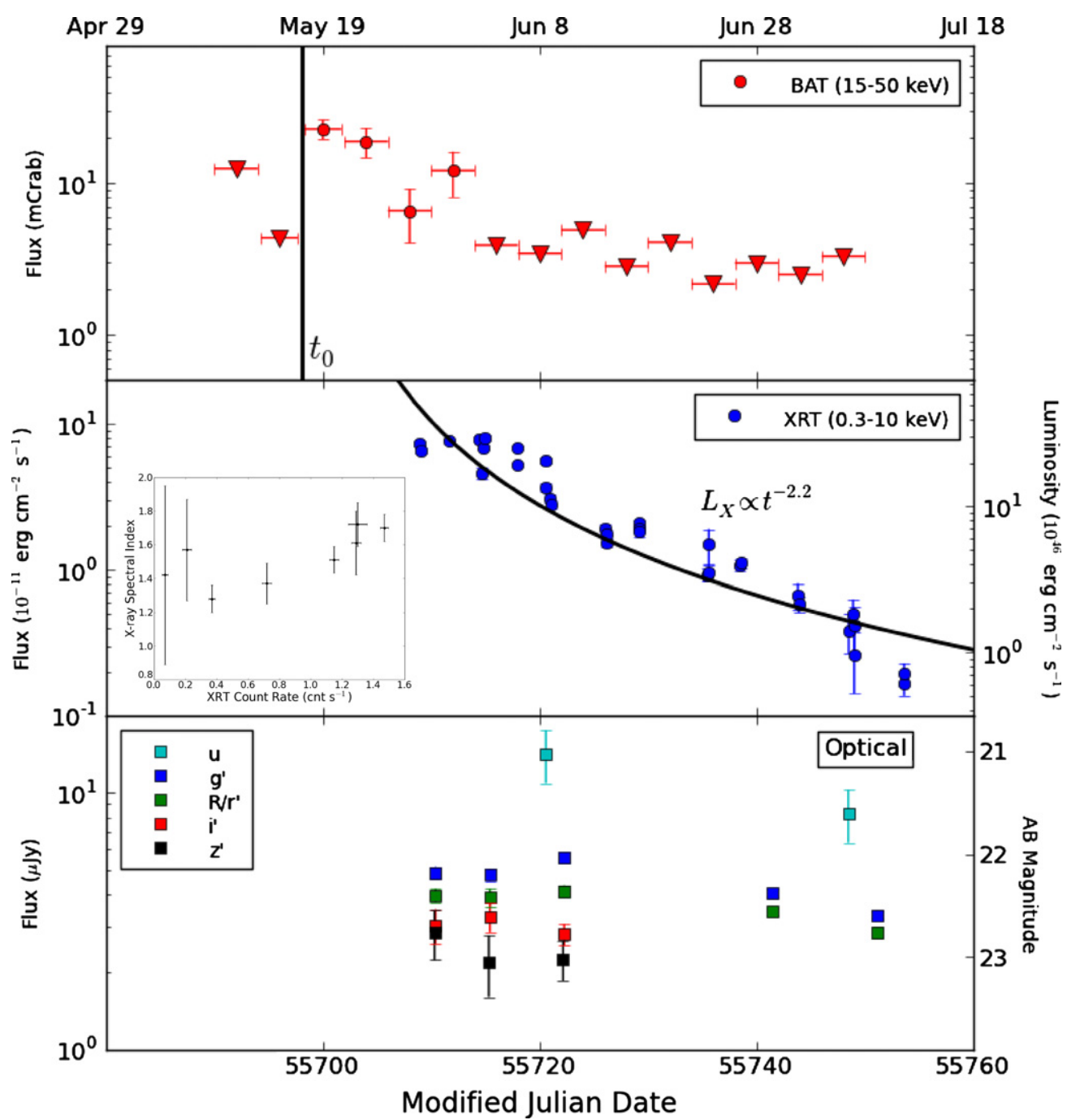

Figure 1. Hard X-ray (15-50 keV), X-ray (0.3-10 keV), and optical light curve of Sw J2058+05. The inset in the X-ray panels shows the derived power-law spectral index $(\Gamma)$ as a function of the $X$-ray count rate (i.e., flux). Inverted triangles represent $3 \sigma$ upper limits.

(A color version of this figure is available in the online journal.)

of the already diverse phenomenology of variability from active galaxies.

Throughout this work, we adopt a standard $\Lambda \mathrm{CDM}$ cosmology with $H_{0}=71 \mathrm{~km} \mathrm{~s}^{-1} \mathrm{Mpc}^{-1}, \Omega_{\mathrm{m}}=0.27$, and $\Omega_{\Lambda}=1-\Omega_{\mathrm{m}}=0.73$ (Spergel et al. 2007). All quoted uncertainties are $1 \sigma(68 \%)$ confidence intervals unless otherwise noted, and UT times are used throughout. Reported magnitudes are in the AB system (Oke \& Gunn 1983).

\section{DISCOVERY AND OBSERVATIONS}

\subsection{Swift-BAT Hard X-Ray Monitor Discovery}

Sw J2058+05 was discovered by the Burst Alert Telescope (BAT; Barthelmy et al. 2005) on the Swift satellite (Gehrels et al. 2004) as part of the hard X-ray monitor's automated transient search. It first reached the $6 \sigma$ discovery threshold in a fourday integration covering the time period 2011 May 17-20, with a $15-50 \mathrm{keV}$ count rate of $0.0044 \pm 0.0006$ counts s${ }^{-1} \mathrm{~cm}^{-2}$ (Krimm et al. 2011). ${ }^{13}$ No significant emission was detected

13 Though not precisely constrained, we hereafter refer to the discovery time $\left(t_{0}\right)$ as 00:00 on 2011 May $17(\mathrm{MJD}=55698)$. from this location after 2011 June 2. The resulting BAT $(15-50 \mathrm{keV})$ light curve is plotted in the top panel of Figure 1.

The observed BAT count rate is too low to measure statistically significant variations on timescales shorter than $4 \mathrm{~d}$ or to constrain the hard X-ray spectrum. A search of transient monitor data on the same $4 \mathrm{~d}$ timescale back to 2005 February shows no previous activity from the source with a $3 \sigma$ count rate limit of $<0.003$ counts $\mathrm{s}^{-1} \mathrm{~cm}^{-2}$.

\subsection{X-Rays}

To confirm this discovery, we requested a Swift target-ofopportunity (ToO) observation, which began at 21:56 on 2011 May 28. Data obtained by the X-ray Telescope (XRT; Burrows et al. 2005) were reduced using the online analysis tools of Evans et al. (2009), and XRT spectra were fitted using xspec12.6. ${ }^{14}$ After correcting the XRT astrometry following Goad et al. (2008), we identified a bright point source at (J2000.0) coordinates $\alpha=20^{\mathrm{h}} 58^{\mathrm{m}} 19^{\mathrm{s}} .85, \delta=+05^{\circ} 13^{\prime} 33^{\prime \prime} .0$, with a $90 \%$ localization radius of 1 1"7. The XRT continued to monitor Sw J2058+05; the resulting 0.3-10 keV light curve is plotted in the middle panel of Figure 1.

\footnotetext{
${ }^{14}$ See http://heasarc.gsfc.nasa.gov/docs/xanadu/xspec/.
} 


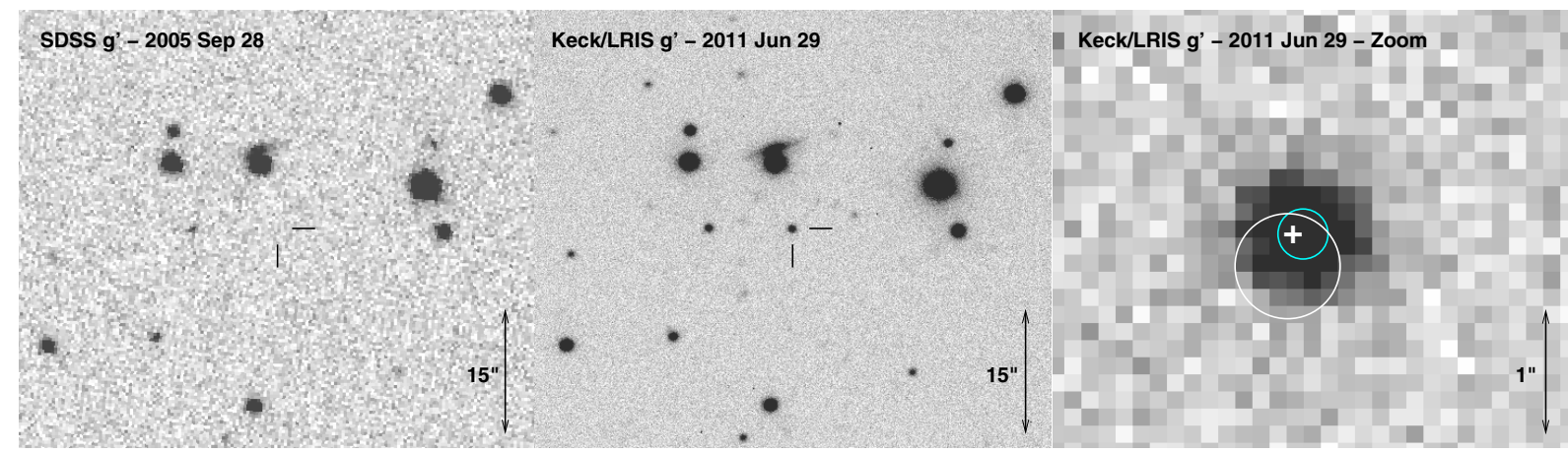

Figure 2. Optical finder chart for the field of Sw J2058+05. Left: pre-outburst SDSS $g^{\prime}$ image. No emission is detected at the position of Sw J2058+05 (indicated with the black tickmarks) to a limiting magnitude of $g^{\prime}>23.5$ mag. Middle: Keck/LRIS $g^{\prime}$ image of the optical transient. The source appears point-like in this image ( 0 '.7 seeing). Right: zoom-in of the Keck/LRIS image of the transient. The uncertainty in the astrometric tie for the optical frame (aligned with respect to 2MASS, which is reprojected onto the ICRS reference system) is indicated by the cyan circle (0!' 2 radius). The Chandra/HRC X-ray localization (based on the native Chandra astrometry, which is generated with respect to the ICRS) is plotted as the white circle (0'4 radius), while the EVLA radio position (50 mas uncertainty) is shown as the white cross. All three positions are entirely consistent. All images are oriented with north up and east to the left.

(A color version of this figure is available in the online journal.)

Superimposed on the secular decline (reasonably well described by a power law: $L_{\mathrm{X}} \propto t^{-2.2}$ ), the X-ray light curve shows some degree of variability on relatively short timescales (compared to the time since discovery). The most significant flaring is detected on 2011 June 2, with a change in flux of a factor of 1.5 occurring on a timescale of $\delta t \approx 10^{4} \mathrm{~s}$. No significant variability is detected on shorter timescales; however, given the signal-to-noise ratio, we are not sensitive to similar variations (i.e., factor of two) on timescales shorter than $\sim 10^{3} \mathrm{~s}$.

The average photon-counting mode spectrum is reasonably well described by an absorbed power law with photon index $\Gamma=1.61 \pm 0.12$ and $N_{\mathrm{H}, \text { host }}=(2.6 \pm 1.6) \times 10^{21} \mathrm{~cm}^{-2}$ $\left(\chi^{2}=65.95\right.$ for 54 degrees of freedom, dof; see Butler \& Kocevski 2007 for details of the spectral analysis). Using this time-averaged spectrum, we find that the peak (unabsorbed) $0.3-10 \mathrm{keV}$ flux is $f_{\mathrm{X}} \approx 7.9 \times 10^{-11} \mathrm{erg} \mathrm{cm}^{-2} \mathrm{~s}^{-1}$. The requirement for absorption in excess of the Galactic value $\left(N_{\mathrm{H}, \mathrm{Gal}}=6.5 \times 10^{20} ;\right.$ Kalberla et al. 2005$)$ is only significant at the $3.2 \sigma$ level. Interestingly, the time-resolved hardness ratio is inversely correlated with the source flux (Figure 1), a behavior commonly seen in Galactic black hole binaries (e.g., Remillard \& McClintock 2006).

To improve the astrometric precision of the X-ray localization, we obtained $\mathrm{ToO}$ observations of Sw J2058+05 with the High Resolution Camera (HRC; Murray et al. 1997) on the Chandra X-Ray Observatory. Observations began at 14:51 on 2011 June 22, for a total live exposure of $5.2 \mathrm{ks}$. Sw J2058+05 is well detected with $(\mathrm{J} 2000.0)$ coordinates $\alpha=20^{\mathrm{h}} 58^{\mathrm{m}} 19.90$, $\delta=+05^{\circ} 13^{\prime} 32^{\prime \prime} .0$, and an astrometric uncertainty of $0^{\prime \prime} \cdot 4$ (Figure 2; the lack of additional point sources in the HRC image precludes us from improving the native Chandra astrometric precision). The X-ray flux shows weak evidence $(1.5 \sigma)$ for a decline over the course of the entire observation, but no statistically significant variability on shorter timescales.

Finally, we note that the location of Sw J2058+05 was observed on 2000 March 16 by the Position Sensitive Proportional Counters on board ROSAT as part of the All-Sky Survey (Voges et al. 1999). No sources are detected in the field to a limit of $f_{\mathrm{X}}(0.1-2.4 \mathrm{keV})<10^{-13} \mathrm{erg} \mathrm{cm}^{-2} \mathrm{~s}^{-1}$, several orders of magnitude fainter than the observed outburst.

\subsection{UV/Optical/NIR Photometry}

Following the discovery of the X-ray counterpart, we initiated a campaign to observe Sw J2058+05 in the ultraviolet (UV), optical, and near-infrared (NIR), with the Swift UltravioletOptical Telescope (UVOT; Roming et al. 2005), the sevenchannel imager GROND (Greiner et al. 2008) mounted on the $2.2 \mathrm{~m}$ telescope at La Silla Observatory, the Low Resolution Imaging Spectrometer (LRIS; Oke et al. 1995) mounted on the $10 \mathrm{~m}$ Keck I telescope, the Wide-Field Camera (WFCAM) on the United Kingdom Infrared Telescope (UKIRT), and the Auxiliary-port Camera (ACAM) on the $4.2 \mathrm{~m}$ William Herschel Telescope. All observations were reduced following standard procedures and photometrically calibrated with respect to the Two Micron All Sky Survey (2MASS; Skrutskie et al. 2006) in the NIR, Sloan Digital Sky Survey (SDSS; Abazajian et al. 2009) in the optical, and following Poole et al. (2008) for the UVOT.

The optical counterpart of Sw J2058+05 was first identified in our GROND imaging obtained on 2011 May 29 at (J2000.0) coordinates $\alpha=20^{\mathrm{h}} 58^{\mathrm{m}} 19.90, \delta=+05^{\circ} 13^{\prime} 32^{\prime \prime} .2$, with an astrometric uncertainty of 0.'2 (radius; Rau et al. 2011a, 2011b). The counterpart appears point-like in all our images, with the tightest constraints provided by our Keck/LRIS images on 2011 Jun 29 (0'7 seeing; Figure 2). Since discovery, we detect statistically significant fading in the bluer filters, but the degree of optical variability is much smaller than that observed in the $\mathrm{X}$-rays. A full listing of our photometry is provided in Table 1, while the light curves are plotted in the bottom panel of Figure 1.

Pre-outburst imaging of the location of Sw J2058+05 was obtained on 2005 September 28 as part of SDSS. No source consistent with the location of Sw J2058+05 appears in the SDSS catalogs. Manually inspecting the images, we calculate $3 \sigma$ upper limits of $u^{\prime}>21.9, g^{\prime}>23.5, r^{\prime}>23.5, i^{\prime}>23.0$, and $z^{\prime}>21.7 \mathrm{mag}$. The lack of a pre-existing counterpart in SDSS is indicative that the observed optical emission is dominated by transient light and not any underlying host galaxy.

\subsection{Optical Spectroscopy}

We obtained a series of optical spectra of Sw J2058+05 on 2011 June 1 with the Deep Imaging Multi-Object Spectrograph (DEIMOS; Faber et al. 2003) mounted on the $10 \mathrm{~m}$ Keck II telescope. The instrument was configured with the 600 lines $\mathrm{mm}^{-1}$ grating, providing spectral coverage over the region $\lambda=4500-9500 \AA$ with a spectral resolution of $3.5 \AA$. The spectra were optimally extracted (Horne 1986), and the rectification and sky subtraction were performed following the 
Table 1

UV/Optical/NIR Observations of Sw J2058+05

\begin{tabular}{|c|c|c|c|c|}
\hline $\begin{array}{l}\text { Date }^{\mathrm{a}} \\
(2011 \mathrm{UT})\end{array}$ & Telescope/Instrument & Filter & $\begin{array}{c}\text { Exposure Time } \\
\text { (s) }\end{array}$ & Magnitude $^{\mathrm{b}}$ \\
\hline May 27.91 & UVOT & $b$ & 2927.3 & $>21.50$ \\
\hline May 28.38 & GROND & $J$ & 720.0 & $>19.6$ \\
\hline May 28.38 & GROND & $H$ & 720.0 & $>19.0$ \\
\hline May 28.38 & GROND & $K$ & 720.0 & $>17.3$ \\
\hline May 29.41 & GROND & $g^{\prime}$ & 3600.0 & $22.55 \pm 0.06$ \\
\hline May 29.41 & GROND & $r^{\prime}$ & 3600.0 & $22.66 \pm 0.07$ \\
\hline May 29.41 & GROND & $i^{\prime}$ & 3600.0 & $22.89 \pm 0.18$ \\
\hline May 29.41 & GROND & $z^{\prime}$ & 3600.0 & $22.91 \pm 0.27$ \\
\hline May 29.41 & GROND & $J$ & 3600.0 & $>21.0$ \\
\hline May 29.41 & GROND & $H$ & 3600.0 & $>20.5$ \\
\hline May 29.41 & GROND & $K$ & 3600.0 & $>18.0$ \\
\hline May 30.58 & UVOT & $u v w 1$ & 2580.1 & $>22.80$ \\
\hline Jun 2.41 & UVOT & uvm2 & 2889.8 & $>23.07$ \\
\hline Jun 3.40 & GROND & $g^{\prime}$ & 1440.0 & $22.57 \pm 0.06$ \\
\hline Jun 3.40 & GROND & $r^{\prime}$ & 1440.0 & $22.68 \pm 0.09$ \\
\hline Jun 3.40 & GROND & $i^{\prime}$ & 1440.0 & $22.81 \pm 0.15$ \\
\hline Jun 3.40 & GROND & $z^{\prime}$ & 1440.0 & $23.20 \pm 0.34$ \\
\hline Jun 3.40 & GROND & $J$ & 1440.0 & $>21.4$ \\
\hline Jun 3.40 & GROND & $H$ & 1440.0 & $>20.9$ \\
\hline Jun 3.40 & GROND & $K$ & 1440.0 & $>18.8$ \\
\hline Jun 5.88 & UVOT & uvw2 & 3059.2 & $>23.37$ \\
\hline Jun 8.57 & UVOT & $u$ & 2258.0 & $21.51 \pm 0.29$ \\
\hline Jun 10.26 & GROND & $g^{\prime}$ & 9600.0 & $22.40 \pm 0.05$ \\
\hline Jun 10.26 & GROND & $r^{\prime}$ & 9600.0 & $22.62 \pm 0.06$ \\
\hline Jun 10.26 & GROND & $i^{\prime}$ & 9600.0 & $22.98 \pm 0.11$ \\
\hline Jun 10.26 & GROND & $z^{\prime}$ & 9600.0 & $23.17 \pm 0.20$ \\
\hline Jun 10.26 & GROND & $J$ & 9600.0 & $>21.9$ \\
\hline Jun 10.26 & GROND & $H$ & 9600.0 & $>21.2$ \\
\hline Jun 10.26 & GROND & $K$ & 9600.0 & $>19.3$ \\
\hline Jun 14.04 & UVOT & uvm 2 & 3113.3 & $>23.13$ \\
\hline Jun 17.11 & UVOT & uvw2 & 2823.8 & $>23.43$ \\
\hline Jun 18.51 & WFCAM & $H$ & 2400.0 & $>20.8$ \\
\hline Jun 18.53 & WFCAM & $K$ & 2400.0 & $>20.9$ \\
\hline Jun 23.54 & UVOT & $u v w 1$ & 2434.2 & $>22.92$ \\
\hline Jun 26.55 & UVOT & uvm2 & 2468.0 & $>23.12$ \\
\hline Jun 29.45 & Keck/LRIS & $g^{\prime}$ & 300.0 & $22.75 \pm 0.04$ \\
\hline Jun 29.45 & Keck/LRIS & $R$ & 280.0 & $22.82 \pm 0.04$ \\
\hline Jul 1.76 & UVOT & $u v w 1$ & 3050.1 & $>23.02$ \\
\hline Jul 6.46 & UVOT & $u$ & 2402.1 & $22.09 \pm 0.29$ \\
\hline Jul 7.00 & UVOT & uvw2 & 430.1 & $>22.21$ \\
\hline Jul 9.15 & WHT & $r^{\prime}$ & 300.0 & $22.96 \pm 0.04$ \\
\hline Jul 9.17 & WHT & $g^{\prime}$ & 300.0 & $23.02 \pm 0.05$ \\
\hline Jul 11.53 & UVOT & uvw2 & 2271.5 & $>23.36$ \\
\hline
\end{tabular}

Notes.

${ }^{\text {a }}$ UT at beginning of exposure.

${ }^{\mathrm{b}}$ Reported magnitudes have not been corrected for Galactic extinction ( $E(B-$ $V)=0.095$ mag; Schlegel et al. 1998). Upper limits represent $3 \sigma$ uncertainties.

procedure described by Kelson (2003). The slit was oriented at the parallactic angle to minimize losses due to atmospheric dispersion (Filippenko 1982).

A portion of the resulting spectrum is plotted in Figure 3. Superimposed on a relatively blue continuum, we identify several strong (rest-frame equivalent width $W_{\mathrm{r}} \gtrsim 1 \AA$ ) absorption features corresponding to $\mathrm{Mg}$ II $\lambda \lambda 2796,2803$, Fe II $\lambda 2600$, Fe II $\lambda 2587$, Fe II $\lambda 2383$, and Fe II $\lambda 2344$ at $z=1.1853 \pm 0.0004$. Given the $U$-band detection $\left(z \lesssim 2\right.$ assuming $\left.\lambda_{\text {Ly } \alpha} \lesssim \lambda_{U}\right)$, we assume that this redshift corresponds to the host galaxy of Sw J2058+05. No other significant features are detected, either in emission or absorption, over the observed bandpass. Specifically, we limit the flux from $[\mathrm{O}$ II $] \lambda 3727$ to be $f<3 \times 10^{-17} \mathrm{erg} \mathrm{cm}^{-2} \mathrm{~s}^{-1}(3 \sigma)$.
A second spectrum was obtained with Keck/LRIS on 2011 June 29 , covering the wavelength range 3500-9500 $\AA$. We find no significant evolution in either the shape of the continuum or the observed absorption features over this time period.

\subsection{Radio}

We observed the location of Sw J2058+05 with the National Radio Astronomy Observatory's Karl G. Jansky Very Large Array (VLA; Perley et al. 2011) ${ }^{15}$ on 2011 June 29.36 and 2011 July 19.39. Observations were conducted in the $\mathrm{C}$ band $(4-8 \mathrm{GHz})$ on June 29 and in the $\mathrm{K}(8-12 \mathrm{GHz})$ and $\mathrm{X}$ (18-26 GHz) bands on July 19. The array was in the A configuration for both epochs, and data were processed using standard routines in the AIPS environment.

Within the EVLA field of view, we detect a single point source with (J2000.0) coordinates $\alpha=20^{\mathrm{h}} 58^{\mathrm{m}} 19.898, \delta=$ $+05^{\circ} 13^{\prime} 32^{\prime \prime} .25$, with an (absolute) astrometric uncertainty of 50 mas. We report the following flux densities: on June 29, $f_{v}(v=4.5 \mathrm{GHz})=0.88 \pm 0.05 \mathrm{mJy}$ and $f_{v}(v=7.9 \mathrm{GHz})=$ $0.84 \pm 0.04 \mathrm{mJy}$; on July $19, f_{v}(v=8.4 \mathrm{GHz})=1.34 \pm$ $0.04 \mathrm{mJy}$ and $f_{v}(v=22.5 \mathrm{GHz})=1.21 \pm 0.15 \mathrm{mJy}$.

The EVLA localization is plotted as a cross in the right panel of Figure 2. Native EVLA astrometry is relative to the ICRS system, which we have also used for the optical (via the 2MASS point source catalog) and X-ray localizations (via the native Chandra pointing solution). All three positions are coincident, implying that the emission we are observing in different bandpasses all results from the same physical source.

This field had been observed previously by the VLA at $1.4 \mathrm{GHz}$, once on 1996 July 8 as part of the NRAO VLA Sky Survey (NVVS; Condon et al. 1998) and three times in 2009 (March 9, March 16, and May 9) as part of the Faint Images of the Radio Sky at Twenty-Centimeters (FIRST; Becker et al. 1995) survey. We inspected the images from these surveys and found no source at the position of Sw J2058+05, with $3 \sigma$ limits of $f_{v}<1.5 \mathrm{mJy}(\mathrm{NVSS})$ and $0.38 \mathrm{mJy}$ (FIRST).

\section{DISCUSSION}

\subsection{Comparison to Sw J1644+57 and Known Classes of Active Galaxies}

In Figure 4, we plot the X-ray and optical luminosity of Sw $\mathrm{J} 2058+05$ at an (observer-frame) time of $\Delta t \approx 12$ days after discovery. For comparison, we also plot analogous measurements for a sample of long-duration GRB X-ray afterglows (at the same epoch post-burst), as well as nearby AGNs, more distant, luminous quasars from SDSS, and some of the most dramatically variable blazars observed while in outburst. With an isotropic $\mathrm{X}$-ray luminosity $L_{\mathrm{X} \text {,iso }} \approx 3 \times 10^{47} \mathrm{erg} \mathrm{s}^{-1}$, Sw J2058+05 is much too luminous at this late time to result from a GRB. Yet with an absolute magnitude of $M_{U} \approx-22.7 \mathrm{mag}$, the observed optical emission is several orders of magnitude underluminous compared with the brightest X-ray quasars and blazars. Even compared with simultaneous X-ray and optical observations of the strongest high-energy flares from some of the best-known blazars (e.g., Markarian 501, Pian et al. 1998; 3C 279, Wehrle et al. 1998; Markarian 421, Fossati et al. 2008), the ratio of $\mathrm{X}$-ray to optical luminosity appears to be quite unique. However, Sw J2058+05 occupies a nearly identical region of this phase space as Sw J1644+57.

\footnotetext{
15 The National Radio Astronomy Observatory is a facility of the National Science Foundation operated under cooperative agreement by Associated Universities, Inc.
} 


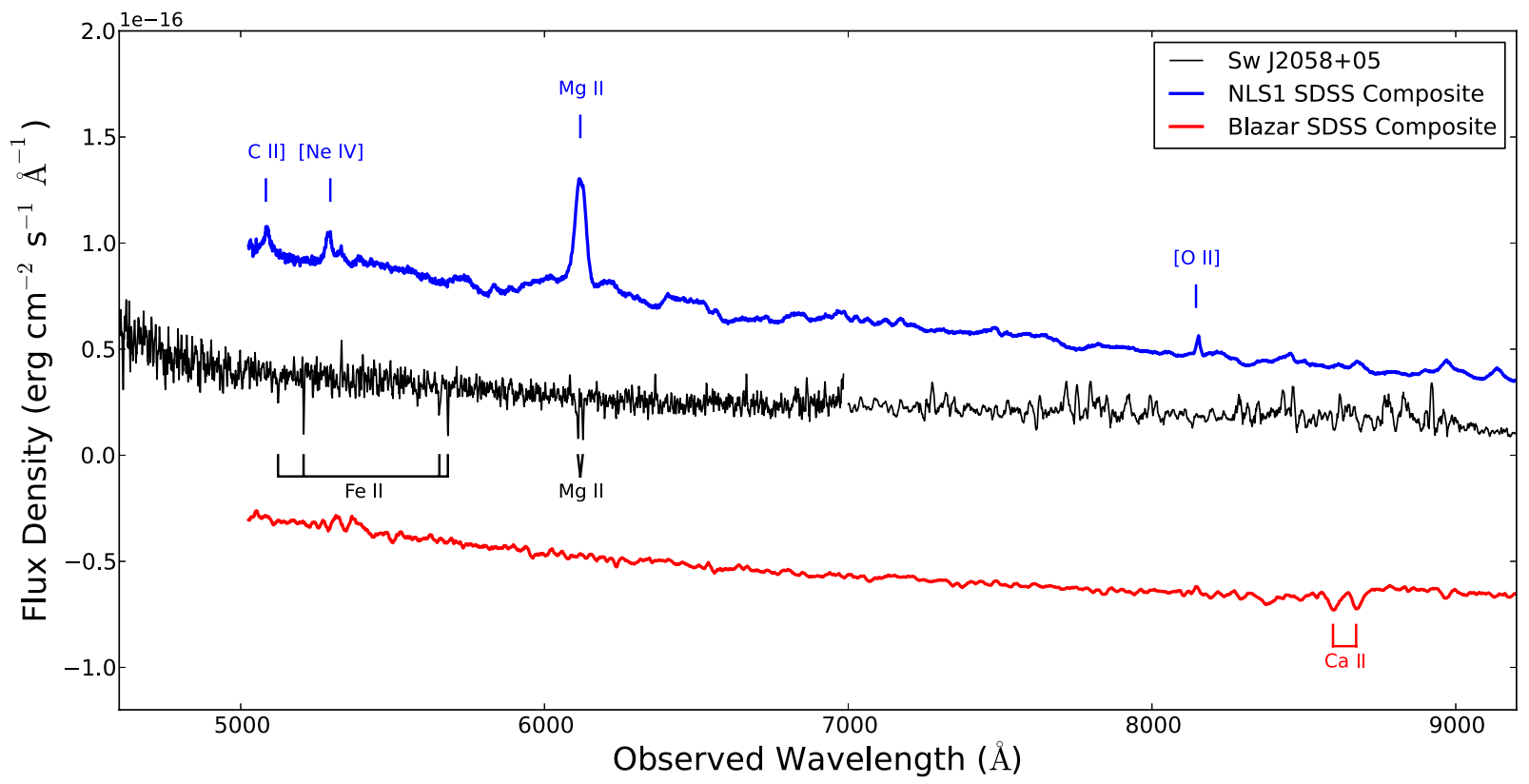

Figure 3. Keck/DEIMOS optical spectrum of Sw J2058+05 (black). The significant absorption features, corresponding to Mg II $\lambda \lambda 2796$, 2803, Fe II $\lambda 2600$, Fe II $\lambda 2587, \mathrm{Fe}$ II $\lambda 2383$, and Fe II $\lambda 2344$ at a common redshift of $1.1853 \pm 0.0004$, are marked. Also plotted is an average of the 478 known NLS1 galaxies (blue) and 68 blazars (red) at $z>0.5$ with spectra in SDSS. All but one of these NLS1 galaxies exhibit broad Mg II emission (as well as weaker C II], [Ne IV], and [O II]), which is entirely lacking from Sw J2058+05. While the blazars (by definition) lack strong emission features, most exhibit Ca II $\lambda \lambda 3934,3968$ absorption, and none have $\mathrm{Mg}$ II detected in absorption. Note that the red-side spectrum of Sw J2058+05 (longward of $\sim 7000 \AA$ ) suffers from relatively poor night-sky subtraction, giving rise to apparent emission lines. Also, the weak emission features near $5300 \AA$ in the blazar spectrum are likely to be noise.

(A color version of this figure is available in the online journal.)

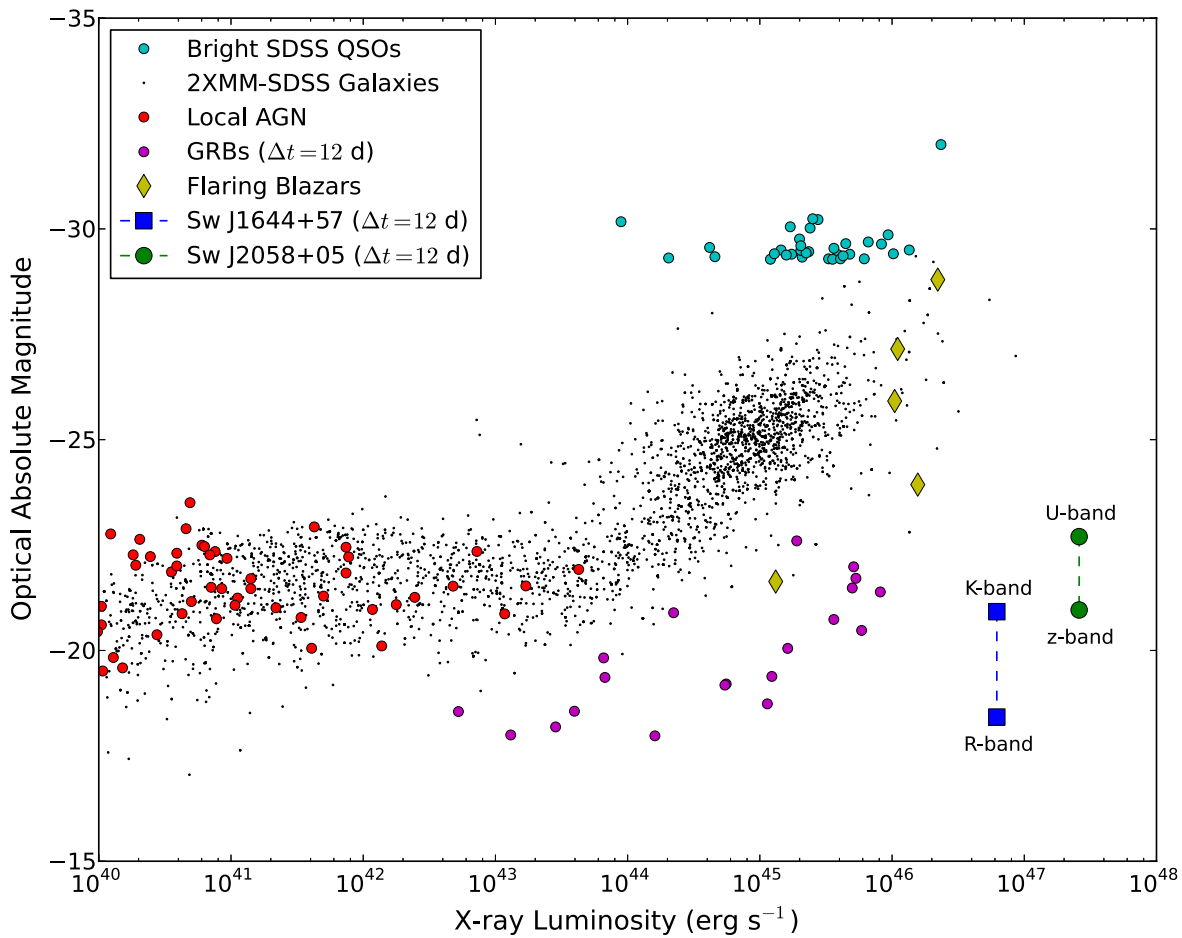

Figure 4. X-ray and optical luminosity of Sw J2058+05, compared with a sample of nearby active galaxies (red circles; Ho 2009), luminous quasars from SDSS (cyan circles), galaxies from SDSS with $X M M$ counterparts (black dots; Pineau et al. 2011), long-duration GRB afterglows (extrapolated to a common epoch of $\Delta t=12$ days after the GRB trigger; Kann et al. 2010; Evans et al. 2009), and high-energy outbursts from known blazars (Markarian 501, Pian et al. 1998; PKS 2155-304, Foschini et al. 2007; PKS 0537-441, Pian et al. 2007; 3C 279, Wehrle et al. 1998; Markarian 421, Fossati et al. 2008). Compared with these sources (and at the same epoch post-discovery as the GRBs), both Sw J2058+05 and Sw J1644+57 exhibit extremely luminous X-ray emission, yet relatively faint optical emission. While for Sw $\mathrm{J} 1644+57$ this is to some extent due to dust extinction in the host galaxy (for which we have not corrected here), the blue UV/optical SED of Sw J2058+05 suggests at most a modest host-galaxy column density. We note that we have not $K$-corrected the absolute magnitudes reported here (aside from cosmological stretch) due to the relatively uncertain intrinsic SEDs. Adapted from Levan et al. (2011).

(A color version of this figure is available in the online journal.) 


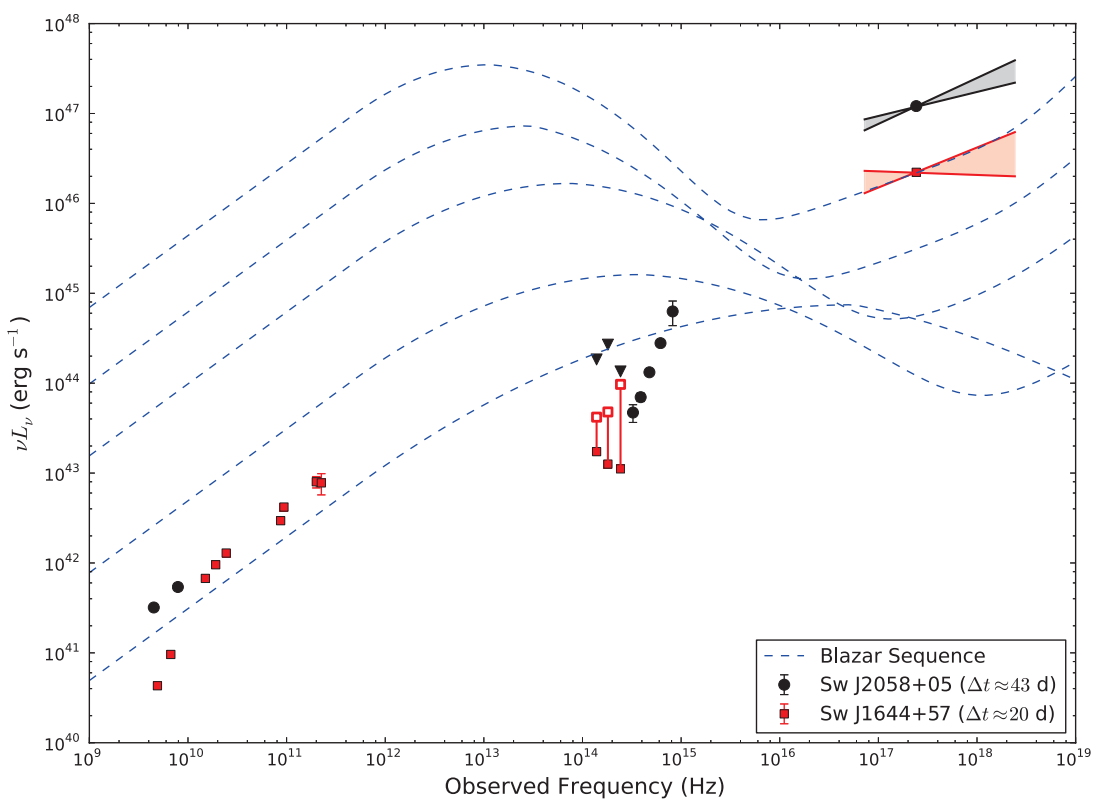

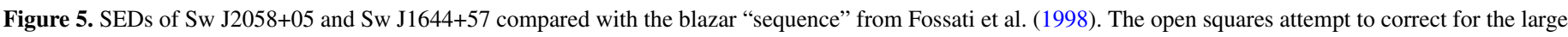

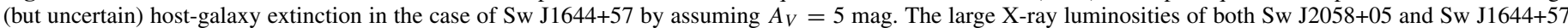

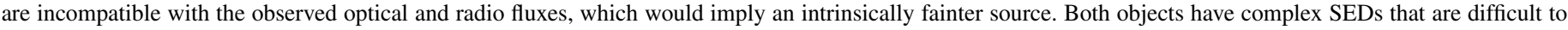
reconcile with only a single emission component (e.g., synchrotron).

(A color version of this figure is available in the online journal.)

This similarity is further reinforced when adding the radio observations to the broadband spectral energy distribution (SED). In Figure 5, we plot the SED of Sw J2058+05 at $\Delta t \approx 43$ days after discovery, compared with analogous measurements for a sample of blazars, the most dramatically variable class of AGNs (and also known sources of relativistic jets; Urry \& Padovani 1995; see Section 3.2). Blazars exhibit a well-defined luminosity "sequence" whereby the lower-frequency maximum of their double-peaked SED occurs at lower energies for more luminous sources (Fossati et al. 1998). While the radio and optical emission from $\mathrm{Sw} \mathbf{J} 2058+05$ are comparable to those seen in low-luminosity blazars, the X-ray emission outshines even the brightest sources of this class. On the other hand, the SED of $\mathrm{Sw} \mathrm{J} 1644+57$, at a time of $\Delta t \approx 20$ days, provides a good match to the observed properties of $\mathrm{Sw} \mathrm{J} 2058+05$ (particularly after correcting for the large but uncertain dust extinction from $\mathrm{Sw}$ J1644+57).

While the sequence plotted in Figure 5 may represent the ensemble properties of blazars as a class, individual sources may exhibit quite different behavior. During an outburst, blazar SEDs can undergo radical changes-for example, as a result of a rapid brightening in 1997, the low-energy (synchrotron) spectral peak in Markarian 501 shifted frequencies by approximately two orders of magnitude (Pian et al. 1998). We therefore also compare the observed SED of Sw J2058+05 with that of several high-energy outbursts from known blazars in Figure 6. Even when compared with these extreme cases, the ratio of the X-ray to optical flux observed in Sw J2058+05 stands out, being similar only to the broadband properties of Sw J1644+57.

The sole remaining defining characteristic of $\mathrm{Sw} \mathrm{J} 1644+57$ is its association with the nucleus of a nonactive galaxy (Levan et al. 2011; Zauderer et al. 2011). At the current time, the observed optical emission from Sw J2058+05 appears to be dominated by the transient, and we have no constraints on the location of the host nucleus from pre-outburst observations. Future high-resolution observations, when the transient light has faded, should be able to constrain the transient-nucleus offset. That said, the detection of strong absorption features indicates that the line of sight penetrates a dense region of the host galaxy (as in the case of some GRBs).

Finally, we utilize the optical spectrum to search for evidence of past AGN activity. Like blazars, NLS1 galaxies are another subclass of AGNs that can sometimes power relativistic jets (e.g., Abdo et al. 2009a, 2009b; Foschini et al. 2011; Section 3.2). Similarly, some NLS1 galaxies have been associated with luminous $\left(L_{\mathrm{X}} \approx 10^{44} \mathrm{erg} \mathrm{s}^{-1}\right) \mathrm{X}$-ray outbursts (e.g., Grupe et al. 1995; Brandt et al. 1995). In the case of Sw $\mathrm{J} 1644+57$, all of the detected emission lines were unresolved, and standard diagnostic diagrams revealed no evidence for ionization from a hidden (i.e., Compton thick) AGN (Levan et al. 2011; Zauderer et al. 2011). The larger redshift precludes such an analysis for $\mathrm{Sw} \mathrm{J} 2058+05$, as the standard diagnostic lines do not fall in the optical bandpass.

However, we have compiled all of the available spectra from SDSS of known NLS1 galaxies (from the catalog of VéronCetty \& Véron 2010) with $z>0.5$ (478 objects) and used these to create an NLS1 template. This composite spectrum is plotted alongside that of Sw J2058+05 in Figure 3. The template exhibits strong, broad emission from C II] $\lambda 2324$, [Ne IV] $\lambda \lambda 2422,2424$, and in particular $\mathrm{Mg}_{\mathrm{II}} \lambda \lambda 2796,2803$. None of these emission lines are detected in our spectrum of Sw J2058+05. In fact, of the 478 known NLS1 galaxies in SDSS with $z>0.5$, all but one have well-detected $(>3 \sigma) \mathrm{Mg}$ II in emission. Unless exceedingly rare, the optical spectrum of Sw J2058+05 suggests that it is not an NLS1 galaxy.

We have furthermore performed a similar analysis with all known BL Lac sources from Véron-Cetty \& Véron (2010) at $z>0.5$ in the SDSS archive (68 sources); the resulting median spectrum is shown in red in Figure 3. By definition, BL Lac objects lack the bright emission features present in the spectra of NLS1 galaxies, and therefore they more closely resemble the observed spectrum of Sw J2058+05. However, BL Lac 


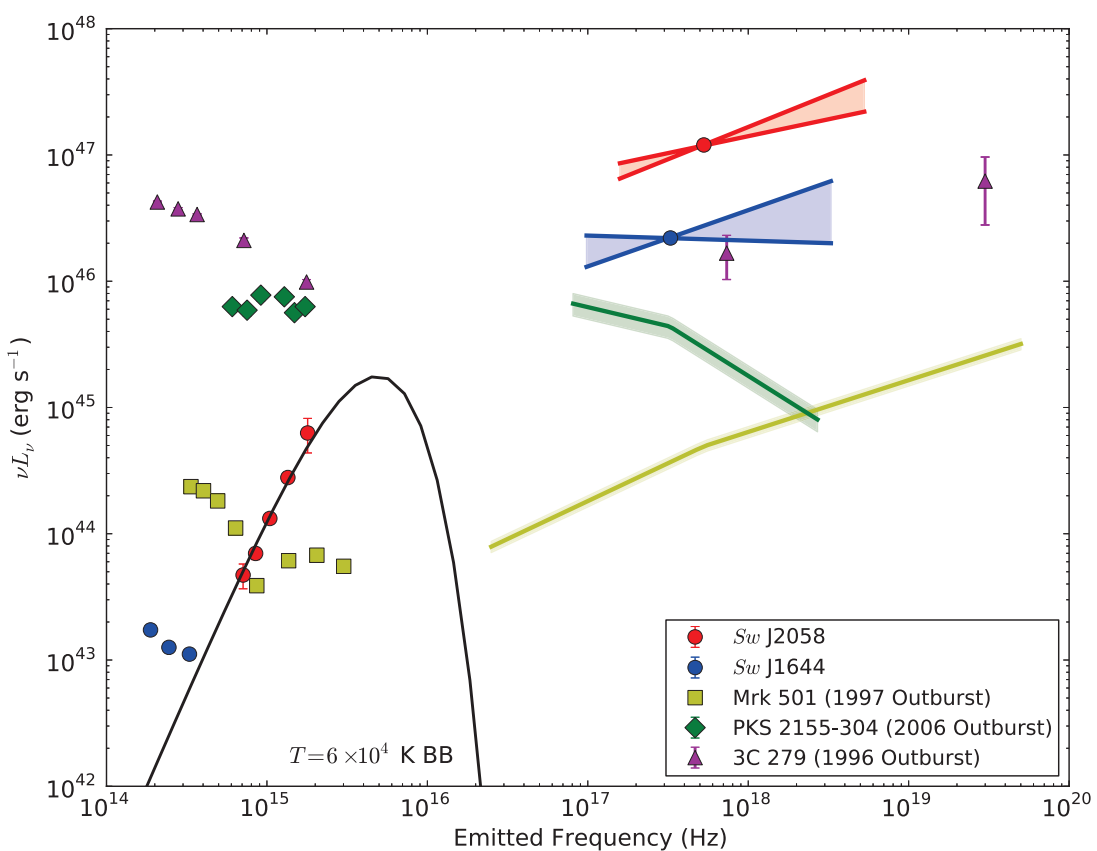

Figure 6. Optical and X-ray SEDs of Sw J2058+05 and Sw J1644+57, compared with some of the most dramatically variable blazars while in outburst (Markarian 501, Pian et al. 1998; PKS 2155-304, Foschini et al. 2007; 3C 279, Wehrle et al. 1998). Unlike any of the other objects plotted here, the rest-frame UV SED of Sw $\mathrm{J} 2058+05$ is well represented by a blackbody by $T \gtrsim 6 \times 10^{4} \mathrm{~K}$ (solid black line).

(A color version of this figure is available in the online journal.)

spectra do typically exhibit Ca II $\lambda \lambda 3934,3968$ in absorption, which is lacking in Sw J2058+05, and not a single BL Lac in the SDSS database exhibits strong Mg II absorption. While we cannot entirely rule out a new mode of variability in a blazar, the unique SED and optical spectrum distinguish Sw J2058+05 from known members of this class as well.

\subsection{Basic Physical Properties}

We have shown in the previous section that $\mathrm{Sw} \mathrm{J} 2058+05$ does not appear to be consistent with any known class of AGNs (particularly blazars and NLS1). Based on the many similarities with Sw J1644+57, we can use the same arguments to derive the basic physical parameters for Sw J2058+05 (e.g., Bloom et al. 2011). From our NIR observations, we derive an upper limit on the host-galaxy absolute $V$-band magnitude of $M_{V} \gtrsim-21 \mathrm{mag}$ (we have used an S0 galaxy template from Kinney et al. 1996 for the $K$-correction based on the lack of emission features in the optical spectrum). Applying the black hole mass vs. bulge luminosity (Magorrian et al. 1998) correlation from Lauer et al. (2007) and assuming a bulge-dominated system, we limit the mass of the putative central black hole to be $M_{\mathrm{BH}} \lesssim 2 \times 10^{8} M_{\odot}$.

Separately, the shortest observed X-ray variability timescale can be used to constrain the central black hole mass using causality arguments (e.g., Campana et al. 2011). For an observerframe variability timescale of $\delta t \lesssim 10^{4} \mathrm{~s}$, we find $M_{\mathrm{BH}} \lesssim$ $5 \times 10^{8} M_{\odot}$. The most dramatic short timescale variability observed from Sw J1644 +57 occurred at $\Delta t \lesssim 10$ days (Bloom et al. 2011), before we commenced X-ray observations of $\mathrm{Sw}$ $\mathrm{J} 2058+05$. But even at late times, the X-ray light curve of Sw J2058+05 exhibits a degree of variability (changes of an order of magnitude on timescales of a few days) not seen in $\mathrm{Sw}$ J2058+05, suggesting an imperfect analogy between these two sources.

Finally, we can constrain the central mass using the black hole fundamental plane, a relationship between mass, X-ray luminosity, and radio luminosity (e.g., Gültekin et al. 2009). Application of this relation requires knowledge of the beaming fraction, for which we adopt a value of $f_{b} \equiv(1-\cos \theta) \approx 10^{-2}$ (see below). Following Miller \& Gültekin (2011), we find $M_{\mathrm{BH}} \approx 5 \times 10^{7} M_{\odot}$; however, we caution that the scatter in this relationship is even larger than that found in the black hole mass versus bulge luminosity relation.

The derived black hole mass, $M_{\mathrm{BH}} \lesssim 10^{8} M_{\odot}$, is of vital importance for two main reasons. First, the tidal disruption of a solar-type star will only occur outside the event horizon for $M_{\mathrm{BH}} \lesssim 2 \times 10^{8} M_{\odot}$. As such, if the origin of the accreting material is indeed the tidal disruption of a nondegenerate star (or more massive object), the mass of the central black hole in the host galaxy of Sw J2058+05 must be sufficiently small. ${ }^{16}$ Future observations of the host, when the optical transient light has faded, should provide a significantly improved estimate of $M_{\mathrm{BH}}$.

Second, for $M_{\mathrm{BH}} \lesssim 10^{8} M_{\odot}$, the observed X-ray emission exceeds the Eddington luminosity. In particular, the peak isotropic $0.3-10 \mathrm{keV}$ X-ray luminosity of $L_{\mathrm{X} \text {,iso }} \approx 3 \times 10^{47} \mathrm{erg} \mathrm{s}^{-1} \mathrm{ex}^{-}$ ceeds the Eddington value by more than an order of magnitude. Given the long-lived nature of the central engine, this suggests that the outflow is likely to be collimated with a beaming factor $f_{b} \lesssim 10^{-1}$ (cf., Socrates 2012). An even larger degree of collimation is required to reconcile the observed rate of these outbursts with theoretical predictions for TDFs (Section 4).

More robust evidence for beaming is provided by the large radio luminosity. It is well known that the brightness temperature of an incoherent radio source cannot exceed $10^{11} \mathrm{~K}$ for extended periods of time (Readhead 1994; Kulkarni et al. 1998). Utilizing this fact, we can derive a lower limit on the angular size of the radio-emitting region, $\theta \gtrsim 14 \mu$ as. Assuming that the outflow

\footnotetext{
16 The limits on the central black hole of the host galaxy of Sw J1644+57 are even more stringent $\left(M \lesssim 10^{7} M_{\odot}\right.$; Levan et al. 2011; Burrows et al. 2011) and may even allow for the disruption of a white dwarf (Krolik \& Piran 2011).
} 
commencement coincides with the hard X-ray discovery, this implies a mean expansion velocity of $\beta \equiv v / c \geqslant 0.88$ or a mildly relativistic expansion with $\Gamma \geqslant 2.1$. Furthermore, the radio emission will be collimated into a viewing angle $\theta \lesssim 1 / \Gamma$ due to relativistic beaming effects.

If we integrate over the X-ray light curve to date (2011 July 20), we find that $\mathrm{Sw} \mathbf{J} 2058+05$ has emitted a total $0.3-10 \mathrm{keV}$ fluence of $S_{\mathrm{X}} \approx 1.0 \times 10^{-4} \mathrm{erg} \mathrm{cm}^{-2}$. If we assume this accounts for $\sim 1 / 3$ of the broadband luminosity, the total isotropic energy release is $E_{\text {iso }} \approx 10^{54} \mathrm{erg}$, comparable to that of Sw J1644+57 at a similar epoch. For a typical radiative efficiency for accretion power $\left(\eta \equiv E_{\text {rad }} / M c^{2} \lesssim 0.1\right)$, the tidal disruption of a solartype star would require a significant beaming correction. If only a small fraction of the available energy is allocated to the observed relativistic component $(\lesssim 1 \%)$, this would imply $f_{b} \lesssim 10^{-3}$.

In addition to the weaker degree of X-ray variability, we wish to draw attention to two other important differences between these sources. Unlike that case of Sw J1644+57, the observed $\mathrm{UV} /$ optical SED of Sw J2058+05 is quite blue. In fact, the restframe UV data for $\mathrm{Sw} \mathrm{J} 2058+05$ can be well fit by a blackbody spectrum (Figure 6), with $T_{\mathrm{BB}} \gtrsim 6 \times 10^{4} \mathrm{~K}, L_{\mathrm{BB}} \gtrsim 10^{45} \mathrm{erg} \mathrm{s}^{-1}$, and $R_{\mathrm{BB}} \gtrsim 10 \mathrm{AU} .{ }^{17}$ The derived blackbody parameters are similar to the thermal emission observed from previous TDF candidates discovered at UV and optical wavelengths (Gezari et al. 2006, 2008, 2009; van Velzen et al. 2011; Cenko et al. 2012), and are strongly suggestive of an accretion disk origin for the optical light. In this sense, Sw J2058+05 may serve as a link between the bright, nonthermal X-rays observed in the relativistic TDF candidates, and the thermal disk emission from "classical" TDFs. Such comparisons were not possible for $\mathrm{Sw}$ $\mathrm{J} 1644+57$ due to dust in the host galaxy.

More importantly, the radio spectral index of Sw J2058+05 appears to be quite flat $\left(f_{v} \propto v^{0}\right)$. This stands in stark contrast to $\mathrm{Sw} \mathbf{J} 1644+57$, which was optically thick $\left(f_{v} \propto v^{1.3}\right)$ up to high frequencies in the days and weeks following discovery (Zauderer et al. 2011). This may simply reflect physical differences in the jet (e.g., microphysics) or its environment (e.g., density), as is suggested by the different extinction properties. However, it may also imply a more extended (and hence longlived) radio source, which would be difficult to reconcile with a tidal disruption origin. Future high-resolution radio interferometry (i.e., VLBI) may be able to resolve this issue.

\section{IMPLICATIONS}

The detection of a second member of this new class of relativistic TDFs would have important implications for the rate of these events. Given the larger redshift of Sw J2058+05, a significantly larger comoving volume (factor of 20) is accessible to detect analogous sources. If we adopt the local density of supermassive black holes from Tundo et al. (2007) $\left(\phi \approx 10^{-3}\right.$ to $\left.10^{-2} \mathrm{Mpc}^{-3}\right)$ and a TDF rate per galaxy of $\sim R \times 10^{-5} \mathrm{yr}^{-1}$ $(R \approx 1-10$; Magorrian \& Tremaine 1999; Donley et al. 2002; Esquej et al. 2007; van Velzen et al. 2011; Bower 2011), the allsky rate of TDFs within this volume is $R \times 10^{5} \mathrm{yr}^{-1}$. Given the long-lived nature of the hard X-ray emission, it seems likely that the Swift-BAT would be capable of detecting similar outbursts within this volume over the entire sky during the course of its seven years of operations to date. Thus, if all TDFs emitted

\footnotetext{
17 Because the observed filters fall on the Rayleigh-Jeans tail, our constraints on the blackbody temperature, luminosity, and radius are essentially lower limits.
}

similarly bright high-energy radiation isotropically, Swift would have detected $\sim 7 R \times 10^{5}$ such outbursts to date.

Given the unique X-ray light curve, it is unlikely we have confused a large number of these events with long-duration GRBs (or, for that matter, other high-energy transients). So that the observed rate is reduced by a factor of $f_{b} \approx 10^{-6}$, commensurate with having only seen two such events, we are therefore left to conclude either (1) the typical beaming fraction of these sources is extremely small $\left(\theta<1^{\circ}\right)$ or (2) only some fraction of TDFs are capable of generating these relativistic outflows. Drawing an analogy with GRBs and core-collapse supernovae, it may be that rapid rotation of the central black hole is required to generate $\mathrm{Sw} \mathrm{J2058+05} \mathrm{like} \mathrm{events.} \mathrm{If} \mathrm{so,}$ TDFs could provide a truly unique diagnostic to constrain the spin of the central black holes in distant galaxies (see also Lei \& Zhang 2011).

Late-time radio observations should be able to distinguish between these two possibilities (Zauderer et al. 2011; Metzger et al. 2012; Berger et al. 2012). Much as has been done with longduration GRBs (Berger et al. 2004; van der Horst et al. 2008), accurate calorimetry can be performed in the nonrelativistic regime when the outflow is spherical, bypassing any concerns about geometric corrections. Separately, the detection of off-axis events could also help address this fundamental issue (Giannios \& Metzger 2011), and such searches will be greatly facilitated by future wide-field radio telescopes (e.g., LOFAR, SKA).

If, on the other hand, it is demonstrated that the emission from Sw J2058+05 was powered by an AGN (e.g., if repeated outbursts are detected in upcoming months and years), this would add yet another example to the already complex taxonomy of active galaxies. The rapid onset of accretion (when compared to the typical system lifetime, $\Delta t_{\mathrm{AGN}} \approx 10^{7} \mathrm{yr}$ ) would likely require some revision of our understanding of how material is channeled into the supermassive black hole and/or the physical processes responsible for launching blazar jets. It would also greatly complicate the search for future TDFs, as standard spectroscopic diagnostic diagrams would appear to be insufficient to distinguish AGNs from normal inactive galaxies.

We thank the Swift PI N. Gehrels and the entire Swift team for their work on the remarkable facilities that enabled the discovery of this event. We thank H. Tananbaum for approving our Chandra ToO request (ObsID 13423), and the entire Chandra staff for the prompt scheduling and execution of these observations. We are grateful to G. Fossati for providing the blazar models in tabular form, D. Poznanski for providing software to calculate the host-galaxy $K$-corrections, and D. Perley for assistance with the reduction of the Keck/LRIS images. We also acknowledge B. Metzger, D. Giannos, and M. Kasliwal for valuable discussions. Public data from the Swift data archive were used for part of this study. Some of the data presented herein were obtained at the W. M. Keck Observatory, which is operated as a scientific partnership among the California Institute of Technology, the University of California and the National Aeronautics and Space Administration; the Observatory was made possible by the generous financial support of the W. M. Keck Foundation. S.B.C. and A.V.F. acknowledge generous financial assistance from Gary \& Cynthia Bengier, the Richard \& Rhoda Goldman Fund, NASA/Swift grants NNX10AI21G and NNX12AD73G, the TABASGO Foundation, and NSF grant AST-0908886.

Facilities: Swift (BAT,XRT,UVOT), UKIRT (WFCAM), Keck:I (LRIS), Keck:II (DEIMOS), Max Planck:2.2m (GROND), ING:Herschel (ACAM) 


\section{REFERENCES}

Abazajian, K. N., Adelman-McCarthy, J. K., Ageros, M. A., et al. 2009, ApJS, 182,543

Abdo, A. A., Ackermann, M., Ajello, M., et al. 2009a, ApJ, 707, 727

Abdo, A. A., Ackermann, M., Ajello, M., et al. 2009b, ApJ, 707, L142

Bade, N., Komossa, S., \& Dahlem, M. 1996, A\&A, 309, L35

Barthelmy, S. D., Barbier, L. M., Cummings, J. R., et al. 2005, Space Sci. Rev., 120,143

Becker, R. H., White, R. L., \& Helfand, D. J. 1995, ApJ, 450, 559

Berger, E., Kulkarni, S. R., \& Frail, D. A. 2004, ApJ, 612, 966

Berger, E., Zauderer, A., Pooley, G. G., et al. 2012, ApJ, 748, 36

Bloom, J. S., Giannios, D., Metzger, B. D., et al. 2011, Science, 333, 203

Bower, G. C. 2011, ApJ, 732, L12

Brandt, W. N., Pounds, K. A., \& Fink, H. 1995, MNRAS, 273, L47

Burrows, D. N., Hill, J. E., Nousek, J. A., et al. 2005, Space Sci. Rev., 120, 165

Burrows, D. N., Kennea, J. A., Ghisellini, G., et al. 2011, Nature, 476, 421

Butler, N. R., \& Kocevski, D. 2007, ApJ, 663, 407

Campana, S., Foschini, L., Tagliaferri, G., Ghisellini, G., \& Covino, S. 2011, GRB Coordinates Network, 1851

Cannizzo, J. K., Troja, E., \& Lodato, G. 2011, ApJ, 742, 32

Cenko, S. B., Bloom, J. S., Kulkarni, S. R., et al. 2012, MNRAS, 420, 2684

Condon, J. J., Cotton, W. D., Greisen, E. W., et al. 1998, AJ, 115, 1693

Donley, J. L., Brandt, W. N., Eracleous, M., \& Boller, T. 2002, AJ, 124, 1308

Esquej, P., Saxton, R. D., Freyberg, M. J., et al. 2007, A\&A, 462, L49

Esquej, P., Saxton, R. D., Komossa, S., et al. 2008, A\&A, 489, 543

Evans, P. A., Beardmore, A. P., Page, K. L., et al. 2009, MNRAS, 397, 1177

Faber, S. M., Phillips, A. C., Kibrick, R. I., et al. 2003, Proc. SPIE, 4841, 1657 Filippenko, A. V. 1982, PASP, 94, 715

Foschini, L., Ghisellini, G., Kovalev, Y. Y., et al. 2011, MNRAS, 413, 1671

Foschini, L., Ghisellini, G., Tavecchio, F., et al. 2007, ApJ, 657, L81

Fossati, G., Buckley, J. H., Bond, I. H., et al. 2008, ApJ, 677, 906

Fossati, G., Maraschi, L., Celotti, A., Comastri, A., \& Ghisellini, G. 1998, MNRAS, 299, 433

Gehrels, N., Chincarini, G., Giommi, P., et al. 2004, ApJ, 611, 1005

Gezari, S., Basa, S., Martin, D. C., et al. 2008, ApJ, 676, 944

Gezari, S., Heckman, T., Cenko, S. B., et al. 2009, ApJ, 698, 1367

Gezari, S., Martin, D. C., Milliard, B., et al. 2006, ApJ, 653, L25

Giannios, D., \& Metzger, B. D. 2011, MNRAS, 416, 2102

Goad, M. R., Tyler, L. G., Beardmore, A. P., et al. 2008, A\&A, 492, 873

Greiner, J., Bornemann, W., Clemens, C., et al. 2008, PASP, 120, 405

Greiner, J., Schwarz, R., Zharikov, S., \& Orio, M. 2000, A\&A, 362, L25

Grupe, D., Beuermann, K., Mannheim, K., et al. 1995, A\&A, 299, L5

Gültekin, K., Cackett, E. M., Miller, J. M., et al. 2009, ApJ, 706, 404

Ho, L. C. 2009, ApJ, 699, 626
Horne, K. 1986, PASP, 98, 609

Kalberla, P. M. W., Burton, W. B., Hartmann, D., et al. 2005, A\&A, 440, 775

Kann, D. A., Klose, S., Zhang, B., et al. 2010, ApJ, 720, 1513

Kelson, D. D. 2003, PASP, 115, 688

Kinney, A. L., Calzetti, D., Bohlin, R. C., et al. 1996, ApJ, 467, 38

Komossa, S., \& Greiner, J. 1999, A\&A, 349, L45

Krimm, H. A., Kennea, J. A., Holland, S. T., et al. 2011, ATel, 3384

Krolik, J. H., \& Piran, T. 2011, ApJ, 743, 134

Kulkarni, S. R., Frail, D. A., Wieringa, M. H., et al. 1998, Nature, 395, 663

Lauer, T. R., Faber, S. M., Richstone, D., et al. 2007, ApJ, 662, 808

Lei, W., \& Zhang, B. 2011, ApJ, 740, 27L

Levan, A. J., Tanvir, N. R., Cenko, S. B., et al. 2011, Science, 333, 199

Magorrian, J., \& Tremaine, S. 1999, MNRAS, 309, 447

Magorrian, J., Tremaine, S., Richstone, D., et al. 1998, AJ, 115, 2285

Maksym, W. P., Ulmer, M. P., \& Eracleous, M. 2010, ApJ, 722, 1035

Metzger, B. D., Giannios, D., \& Mimica, P. 2012, MNRAS, 420, 3528

Miller, J. M., \& Gültekin, K. 2011, ApJ, 738, L13

Murray, S. S., Chappell, J. H., Kenter, A. T., et al. 1997, Proc. SPIE, 3114, 11

Oke, J. B., Cohen, J. G., Carr, M., et al. 1995, PASP, 107, 375

Oke, J. B., \& Gunn, J. E. 1983, ApJ, 266, 713

Perley, R. A., Chandler, C. J., Butler, B. J., \& Wrobel, J. M. 2011, ApJ, 739, L1

Pian, E., Romano, P., Treves, A., et al. 2007, ApJ, 664, 106

Pian, E., Vacanti, G., Tagliaferri, G., et al. 1998, ApJ, 492, L17

Pineau, F.-X., Motch, C., Carrera, F., et al. 2011, A\&A, 527, A126

Poole, T. S., Breeveld, A. A., Page, M. J., et al. 2008, MNRAS, 383, 627

Rau, A., Greiner, J., \& Olivares, F. 2011 a, ATel, 3390

Rau, A., Greiner, J., Schady, P., et al. 2011b, ATel, 3425

Readhead, A. C. S. 1994, ApJ, 426, 51

Rees, M. J. 1988, Nature, 333, 523

Remillard, R. A., \& McClintock, J. E. 2006, ARA\&A, 44, 49

Renzini, A., Greggio, L., di Serego Alighieri, S., et al. 1995, Nature, 378, 39

Roming, P. W. A., Kennedy, T. E., Mason, K. O., et al. 2005, Space Sci. Rev., 120,95

Schlegel, D. J., Finkbeiner, D. P., \& Davis, M. 1998, ApJ, 500, 525

Skrutskie, M. F., Cutri, R. M., Stiening, R., et al. 2006, AJ, 131, 1163

Socrates, A. 2012, ApJ, in press (arXiv:1105.2557)

Spergel, D. N., Bean, R., Doré, O., et al. 2007, ApJS, 170, 377

Tundo, E., Bernardi, M., Hyde, J. B., Sheth, R. K., \& Pizzella, A. 2007, ApJ, 663,53

Urry, C. M., \& Padovani, P. 1995, PASP, 107, 803

van der Horst, A. J., Kamble, A., Resmi, L., et al. 2008, A\&A, 480, 35

van Velzen, S., Farrar, G. R., Gezari, S., et al. 2011, ApJ, 741, 73

Véron-Cetty, M.-P., \& Véron, P. 2010, A\&A, 518, A10

Voges, W., Aschenbach, B., Boller, Th., et al. 1999, A\&A, 349, 389

Wehrle, A. E., Pian, E., Urry, C. M., et al. 1998, ApJ, 497, 178

Zauderer, B. A., Berger, E., Soderberg, A. M., et al. 2011, Nature, 476, 425 